\title{
Bi-Plasma Interactions on Femtosecond Time-Scales
}

\author{
Emily A. Sprague \\ Office of Science, Science Undergraduate Laboratory Internship Program \\ University of Illinois \\ Urbana-Champaign, IL \\ SLAC National Accelerator Laboratory \\ Menlo Park, CA
}

July 21,2010

Prepared in partial fulfillment of the requirements of the Office of Science, Department of Energy's Science Undergraduate Laboratory Internship under the direction of Aaron Lindenberg at the PULSE Institute for Ultrafast Energy Science, Stanford Linear Accelerator Center.

Participant:

Signature

Research Advisor:

Signature 


\section{TABLE OF CONTENTS}

$\begin{array}{ll}\text { Abstract } & \text { iii }\end{array}$

$\begin{array}{ll}\text { Introduction } & 1\end{array}$

Materials and Methods $\quad 4$

$\begin{array}{ll}\text { Results } & 6\end{array}$

$\begin{array}{lr}\text { Conclusions } & 8\end{array}$

$\begin{array}{lr}\text { Acknowledgments } & 9\end{array}$

$\begin{array}{ll}\text { References } & 10\end{array}$

$\begin{array}{ll}\text { Figures } & 11\end{array}$ 


\begin{abstract}
Bi-plasma interactions on femtosecond time-scales. EMILY SPRAGUE (University of Illinois, Urbana-Champaign, IL 61801) AARON LINDENBERG (PULSE Institute for Ultrafast Energy Science, Stanford Linear Accelerator Center, Stanford, CA 94025)

Ultrafast $\mathrm{THz}$ radiation has important applications in materials science studies, such as characterizing transport properties, studying the vibrational response of materials, and in recent years, controlling materials and elucidating their response in intense electromagnetic fields. $\mathrm{THz}$ fields can be generated in a lab setting using various plasma-based techniques. This study seeks to examine the interaction of two plasmas in order to better understand the fundamental physics associated with femtosecond filamentation processes and to achieve more efficient $\mathrm{THz}$ generation in a lab setting. The intensity of fluorescence in the region of overlap was measured as a function of polarization, power, and relative time delay of the two plasma-generating laser beams. Results of time dependent intensity studies indicate strikingly similar behaviors across polarizations and power levels; a sudden intensity spike was observed at time-zero, followed by a secondary maxima and subsequent decay to the initial plasma intensity. Dependence of the intensity on the power through either beam arm was also observed. Spectral studies of the enhanced emission were also carried out. Although this physical phenomenon is still not fully understood, future studies, including further spectral analysis of the fluorescence overlap, could yield new insight into the ultrafast processes occurring at the intersection of femtosecond filaments, and would provide a better understanding of the mechanisms for enhanced $\mathrm{THz}$ production.
\end{abstract}




\section{INTRODUCTION}

Terahertz radiation comprises electro-magnetic waves with frequencies on the order of

$10^{12} \mathrm{~Hz}$. Their low photon energies $(\sim 25 \mathrm{meV})$ allow them to penetrate non-conducting material or objects. Additionally, $\mathrm{THz}$ fields, as a form of non-ionizing radiation, could potentially replace X-rays as a structural probe. Applications of this type of radiation extend to areas such as medical imaging, security, and atomic spectroscopy.

Researchers at SLAC National Accelerator Laboratory's PULSE Institute are currently working to produce intense half-cycle terahertz pulses which turn on and off in hundreds of femtoseconds. This radiation will potentially be used in all-optical studies of materials as it allows biasing in materials without the use of electrodes. This experiment examines the interaction of two plasmas generated by femtosecond pulses in order to better understand unexplored processes at the intersection of two femtosecond filaments, and how to make more efficient strong-field, intense, terahertz beams in a lab-scale situation.

\section{i. Terahertz Production}

To date, there are four broad categories of plasma-based $\mathrm{THz}$ generation techniques [1]. The first technique utilizes the radial acceleration of ionized electrons in a radial intensity gradient produced by an optical beam. Due to the ponderomotive force, this results in a conical THz emission at a given angle to the pulse propagation. A second method uses focused fewcycle pulses with under 10 fs durations to generate $\mathrm{THz}$ emission [1].

Of all the techniques, most attention has been directed toward the AC-bias and DC-bias methods. A typical experimental setup for both the AC-bias and DC-bias method consists of an incoming beam that is then split by a beam-splitter into two beam arms. In the Auston switch 
approach, a DC-bias is applied to the plasma which generates a transverse external polarization, which results in around an order-of-magnitude $\mathrm{THz}$ field increase. The AC-bias approach also produces a transverse polarization, but without electrodes. Instead, a superposition of fundamental and second-harmonic pulse fields is used in order to produce a time-dependent current in the plasma region. Mixing the fundamental and second-harmonic fields allows for efficient $\mathrm{THz}$ generation; because one of the laser frequencies is an integer multiple of the other one, the net current as a result does not vanish over an entire set of cycles [2]. Consequently, by utilizing a focused, short pulse and four-wave mixing of the fundamental and second harmonic waves, electric fields with higher amplitudes can be generated [3]. Our experiment grew out of observations we made while employing the AC-bias approach.

There are several factors that influence the optimization of $\mathrm{THz}$ generation in the AC-bias approach. In order for the second harmonic wave to produce a maximum polarization asymmetry, it must possess a certain phase shift relative to the fundamental harmonic wave. The exact temporal overlap of the two pulses also contributes to maximum $\mathrm{THz}$ generation.

Additionally, the polarization of the fundamental and second-harmonic waves heavily impacts the strength and polarization of the resulting $\mathrm{THz}$ wave [1]. The amplitude and the polarization of the resulting electrical current depends directly on the relative phase between the fundamental and second harmonic waves [4].

\section{ii. Femtosecond Laser Pulses}

Ultrafast pulses are commonly created by using mode-locked oscillators and are used in the production of plasma. One of the most-widely used tunable femtosecond laser sources is the Ti:sapphire amplifier. The Ti:sapphire crystal lases between the wavelengths of 
$680 \mathrm{~nm}$ to $1130 \mathrm{~nm}$, which is the largest tuning range of any laser in this class. The shape of each of those femtosecond pulses can be described using a Gaussian function.

Fourier analysis shows that femtosecond laser pulses can only be generated with a broad spectral bandwidth. The minimum "transform-limited" duration of a pulse can be calculated for a given spectrum and central wavelength. To optimize a femtosecond pulse, the bandwidth and chirp must be considered. Both minimal chirp and large bandwidth are considered ideal. The chirp of a pulse refers to its spectral content and the bandwidth refers to the range of frequency. In this experiment, we used $1 \mathrm{~mJ}$ pulses centered at $800 \mathrm{~nm}$ with a pulse duration measured to be 44 fs using single-shot autocorrelation.

\section{iii. Kerr Lens Mode-locking}

A free-running laser simultaneously oscillates over all of the resonant frequencies in a given cavity producing continuous wave radiation at a narrow wavelength. To create trains of very short and intense light pulse, the laser is mode-locked. Mode-locking forces all of the modes, which are a set of frequencies, to have equal phase by introducing a disturbance to the system. This results in the constructive interference of all the different frequency waves at one point, producing a very intense and extremely short spike or a 'pulse'. There are two methods of mode-locking femtosecond lasers: passive mode-locking and active mode-locking. Active mode-locking requires modulation of the laser that is generated from an external clock source; however, passive mode-locking does not require an external clock, instead it requires the movement of a non-linear device within the cavity (e.g. moving a prism in or out of the cavity). 


\section{iv. Plasma Filamentation}

Plasma, considered to be the most abundant state of matter, comprises ionized gas particles. Due to the presence of an abundance of charge carriers, plasma interacts easily with surrounding electromagnetic fields. Plasma can be formed through photoionization where photons from an external source, such as a laser, are absorbed by a gas, such as air, resulting in cations and emitted electrons. The intensity of $\mathrm{THz}$ radiation generated from plasma formation is dependent on the length and density of the plasma. Additionally, the beam profile and divergence of the THz radiation depend on the focal length of the lenses used [1].

\section{MATERIALS AND METHODS}

\section{i. Setup}

Figure 1 shows the setup used consisting of lenses, mirrors, and beam-splitters. The lab setup mirrors previous bi-plasma crossing experiments performed in our lab [5]. $44 \mathrm{fs,}$ $800 \mathrm{~nm}$ laser pulses generated by a passively mode-locked Ti:sapphire laser were split by a polarizing beam-splitter, which produced a horizontally (or p) polarized beam down arm 1 and a vertically (or s) polarized beam down arm 2 . One of the laser pulses was focused by a $\mathrm{f}=100 \mathrm{~mm}$ lens, which generated a short plasma (P1) in air. The other laser pulse was focused by a f=200 $\mathrm{mm}$ lens to create a longer orthogonal plasma (P2) that crosses the beam path of the first plasma. A delay stage controlled the relative distance and the time between the arrival of the two plasmas. A CCD camera, with a lens of working distance $\sim 5 \mathrm{~mm}$, mounted about 1.5 inches above the intersection of the plasma, was used to image the bi-plasma fluorescence phenomenon. Additionally, ND filters were placed in between the plasma region and the camera lens in order to increase the contrast ratio and prevent camera saturation between the plasma and background pixels. 


\section{ii. Studies}

Much of the fundamental physics regarding the processes that occur within this plasma intersection remain unclear. At the intersection, for at least 20 picoseconds after timezero, the photons from the lagging laser beam interact with the preformed plasma from the earlier laser beam. When both incoming beams spatially and temporally overlap, also referred to as time -zero, the resulting interaction produces an increase of intensity, surprisingly. We hypothesize that this is due to one of two effects: scatter during the reflected plasma lifetime or enhanced tunneling ionization.

Several experiments were set up to better understand the plasma in this area of overlap. In all scans, the dependence of the intensity of the fluorescent light as a function of time was studied. We varied the relative angle of polarization of the two incoming beams. Three configurations were used: s-s polarized, s-p polarized, and p-p polarized, as shown in Figure 2. To change the polarization of a beam path from s to $\mathrm{p}$ polarized, or vise versa, a half-wave plate was used. In the s-s polarization tests, both beam 1 and beam 2 were polarized in two different horizontal directions within the plane of propagation. For the s-p polarization, only beam 1 was horizontally polarized while beam 2 was vertically polarized. The p-p polarization contained both vertically polarized beams.

Additionally, a separate study was conducted using a blue bandpass filter, which blocks $800 \mathrm{~nm}$ and surrounding wavelengths of light, in order to characterize the properties of the fluorescence in the region of overlap. Similar time dependent intensity tests were performed using this setup in an attempt to distinguish between two known effects: scattering or tunneling ionization. 


\section{iii. Data Analysis}

Data in this experiment was acquired using a home-built setup. For time dependent intensity studies, the camera images of the plasmas were analyzed using image processing techniques with the programs ImageJ and Matlab. Peak intensity data were averaged over 400-500 pixels at the plasma intersection, where each pixel had a diameter of 2.6 um. Background noise was subtracted from each pixel in the plasma intersection. Intensities were plotted as a function of increasing time.

\section{RESULTS}

Plasma filamentation and the fluorescent area of bi-plasma intersection were observed in the lab (Figure 3). Figure 4 shows three primary events in this plasma-crossing experiment: before time-zero, at time-zero or the primary intensity maxima, after time-zero at the secondary intensity maxima. A sudden and very dramatic fluorescence was observed at time-zero when both plasmas intersected, as can be seen in Figure 4. This peak intensity in the overlap region at time-zero was followed by a secondary burst of intensity about 30 ps later. Figure 5 shows the time-resolved traces for the peak intensities of the s-s, s-p, and p-p polarization sets. Observing across polarization sets, the peak intensity and point of decay consistently occur at the same time values regardless of the polarization or power of the incoming beams: 135 counts for peak intensity and $\sim 177$ counts for decay. Additionally, the time required for the intensity of the second peak to decay to the initial intensity was constant across all polarizations ( $\sim 50$ steps $)$. All power levels and polarization sets experienced a full decay back to the starting intensities. No valuable data was obtained below a power of $250 \mathrm{~mW}$ in either beam path. The peak intensity at a given power level was always strongest for s-p polarizations and weakest for p-p polarizations. 
The data exhibits interesting trends that depend on the relative power of either beam path. For example, the slope of the decay seems to decrease with decreasing power in the stationary arm. Also, the ratio of the peak intensity to background level intensity seems to decrease with increasing power in the delay arm (beam 1). This trend can be seen in Figure 6 for both the s-p and p-p polarizations; however, when examining the data for the s-s polarization, we noticed a direct rather than an inverse relationship exists between the intensity ratio and the power through the delay arm. In addition, similar trends exist in the ratio of the intensity of the secondary maxima to the background level.

Studies concerning the spectral properties of the fluorescent overlap region yielded interesting results. As shown in Figure 7, the time dependent intensity traces for data taken, with and without a blue bandpass filter, appear to be very similar in both overall shape and absolute values. Figure 8 displays camera images taken without any filter (ND or bluepass). Interestingly, secondary plasmas can be seen along the corners of the overlapping region as indicated in red. These secondary plasmas produce the same intensity level as the region of biplasma overlap. Additional interesting behavior can be seen in Figure 9, in which the fluorescent region of overlap appears to rotate clockwise through time. 


\section{CONCLUSIONS}

Although the data in this experiment is still preliminary, we can still infer some interesting conclusions propose direction for future studies. The images in Figure 9 show a wavefront disturbance. The time dependent intensity trace displays identical general behavior regardless of polarization and power through either beam arm. Although relative intensities vary among tests, the data always exhibits a dramatic spike at time-zero, followed by a secondary maxima and subsequent decay back to the starting intensity. The origin of this unexpected spike in intensity at time-zero is still not fully understood; however, data from the blue bandpass filter tests indicate that the processes governing the bi-plasma overlap likely result from an enhanced tunneling ionization process as opposed to scattering. Because the two traces (representing data taken with a blue bandpass filter and that taken without a blue bandpass filter) are almost identical, we can infer that the spike in intensity is not primarily due to $800 \mathrm{~nm}$ light, as the blue bandpass filter acts to block wavelengths of around $800 \mathrm{~nm}$. Additionally, images in Figure 8 indicate that the observed secondary plasmas are likely not due to fluorescence, as shown by the disappearance of these secondary plasmas upon the addition of a blue bandpass filter.

An alternate possibility that could account for this fluorescence is Raman scattering [6]. In this study, Zhao, Witt, and Gordon report energy transfer between two laser beams intersecting at an acute angle. They observe an angular dependence of the fluorescent plasma intersection and a disappearance of the effect when the two incoming beams are orthogonal. Although intriguing, this explanation does not quite fit with the results presented in our study.

Looking at the power dependent polarization data in Figure 6, the linear relationship is not quite understood. The unexpected behavior of the s-s polarization in comparison to the s-p 
and p-p polarization plots could perhaps be, in part, due to the constructive interference of both incoming s-polarized fields. Of the three polarization sets, the s-s combination is the only set in which the two incoming fields interfere constructively, which could perhaps give rise to this trend reversal in Figure 6.

In conclusion, by crossing two laser-generated plasma filaments we are able to observe fluorescence in the region of overlap perhaps due to the tunneling ionization effect. This fluorescent behavior through time was consistent across all polarizations and power levels. Future studies will involve detailed spectral analysis of this region using a fiber-coupled spectrometer to obtain the exact distribution and composition of the fluorescent light. By understanding how multiple plasmas interact, we hope to not only better understand ultrafast filamentation processes but also develop more efficient plasma-based methods for generating $\mathrm{THz}$ fields in a lab situation.

\section{ACKNOWLEDGEMENTS}

I would like to thank my mentor Aaron Lindenberg for direction and support. I would also like to thank Dan Daranciang, whom I worked alongside, for his assistance and encouragement. Additionally I would like to thank Haidan Wen for his help. Special thanks to SLAC National Accelerator Laboratory and the United States Department of Energy for this research experience. 


\section{REFERENCES}

[1] Thomson, M.D., Kreß M., Loffler T., and Roskos H.G., Broadband THz emission from gas plasmas induced by femtosecond optical pulses: From fundamentals to applications, Laser and Photonics Reviews, 1, No. 4, 349-368 (2007).

[2] Kim K.Y., Glownia J.H., Taylor A.J., and Rodriguez G., Terahertz emission from ultrafast ionizing air in symmetry-broken laser fields, Optics Express, 15, No. 8, 4577 (2007).

[3] Bartel T., Gaal P., Reimann K., Woerner M., and Elsaesser T., Generation of single-cycle THz transients with high electric-field amplitudes, Optics Letters, 30, No. 20, 2805 (2005).

[4] Kim K.Y., Taylor A.J., Glownia J.H., AND Rodriguez G., Coherent control of terahertz supercontinuum generation in ultrafast laser-gas interactions, Nature Photonics, 2, 605 (2008). [5] Wen H., Daranciang D., Lindenberg A.M., High-speed all-optical terahertz polarization switching by a transient plasma phase modular, Applied Physics Letters, 96, 161103 (2010).

[6] Zhao Y., Witt T.E. , and Gordon R. J., Efficient Energy Transfer between Laser Beams by Stimulated Raman Scattering, Physical Review Letters, 103, 173903 (2009). 


\section{FIGURES}

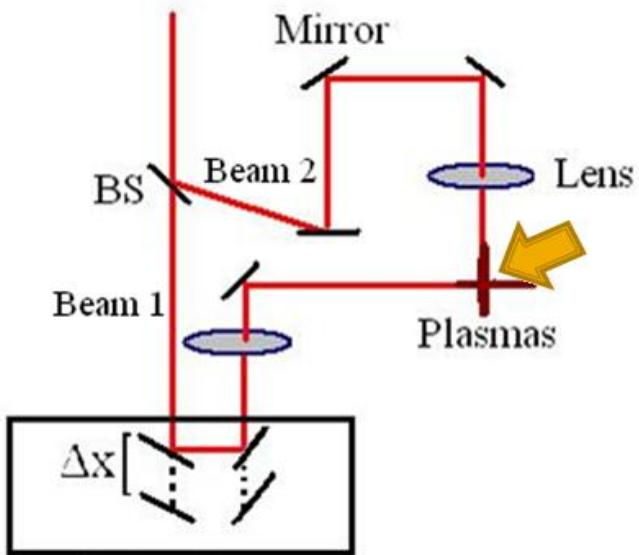

Delay Stage

(a)

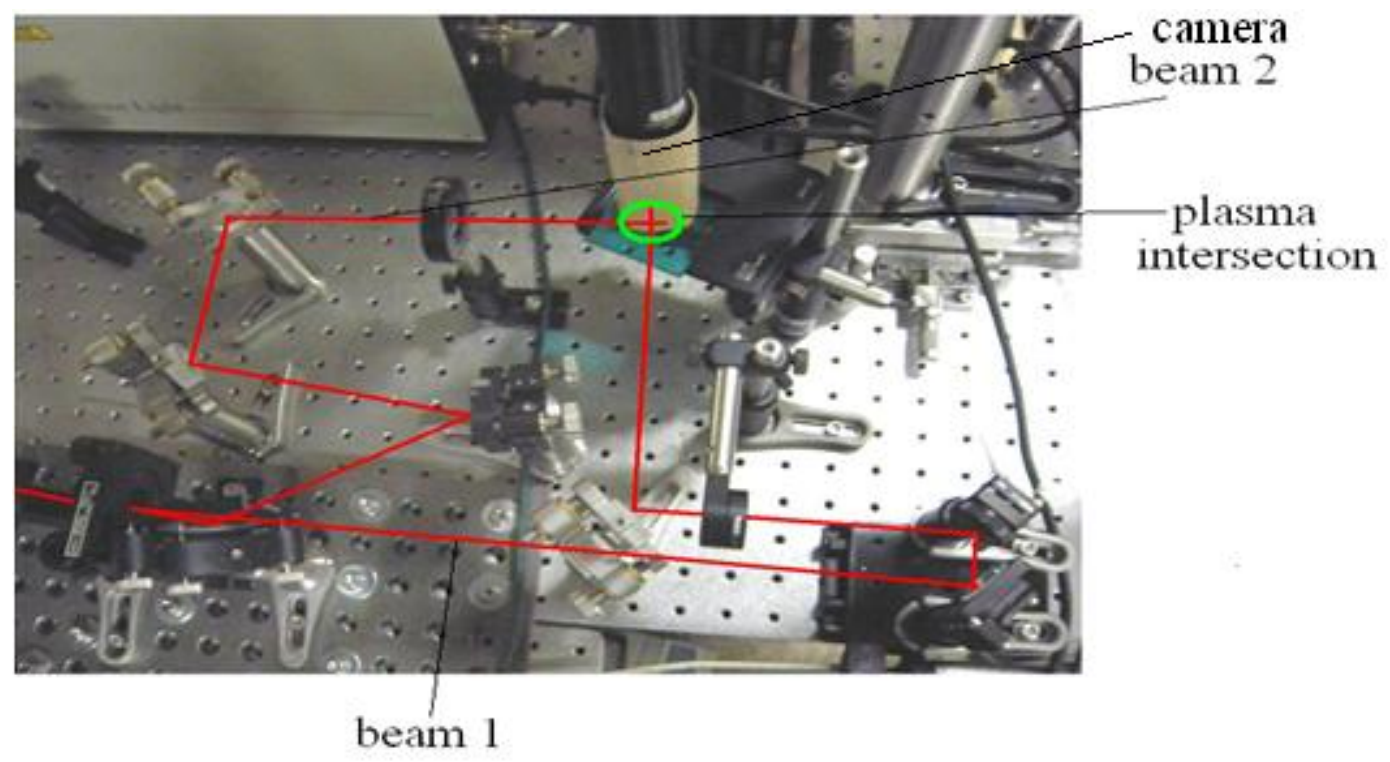

(b)

Figure 1. (a) Schematic of setup consisting of a polarizing beam-splitter, mirrors, lenses, and a delay stage. Yellow arrow indicates position at which bi-plasma phenomenon was observed and placement of CCD camera. (b) Photograph of lab setup with laser beam paths shown in red and plasma intersection in green. 


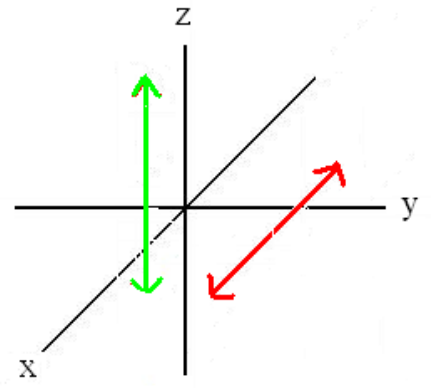

a)

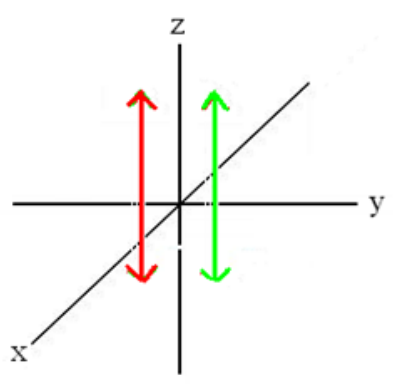

b)

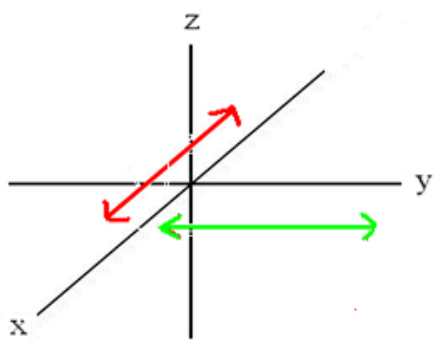

c)

Figure 2. Visual representation of polarization sets for beam 1 and beam 2. a) s-p polarization with one beam vertically polarized and the other beam horizontally polarized. b) s-s polarization with both beams vertically polarized. c) p-p polarization with both beams horizontally polarized in different directions.

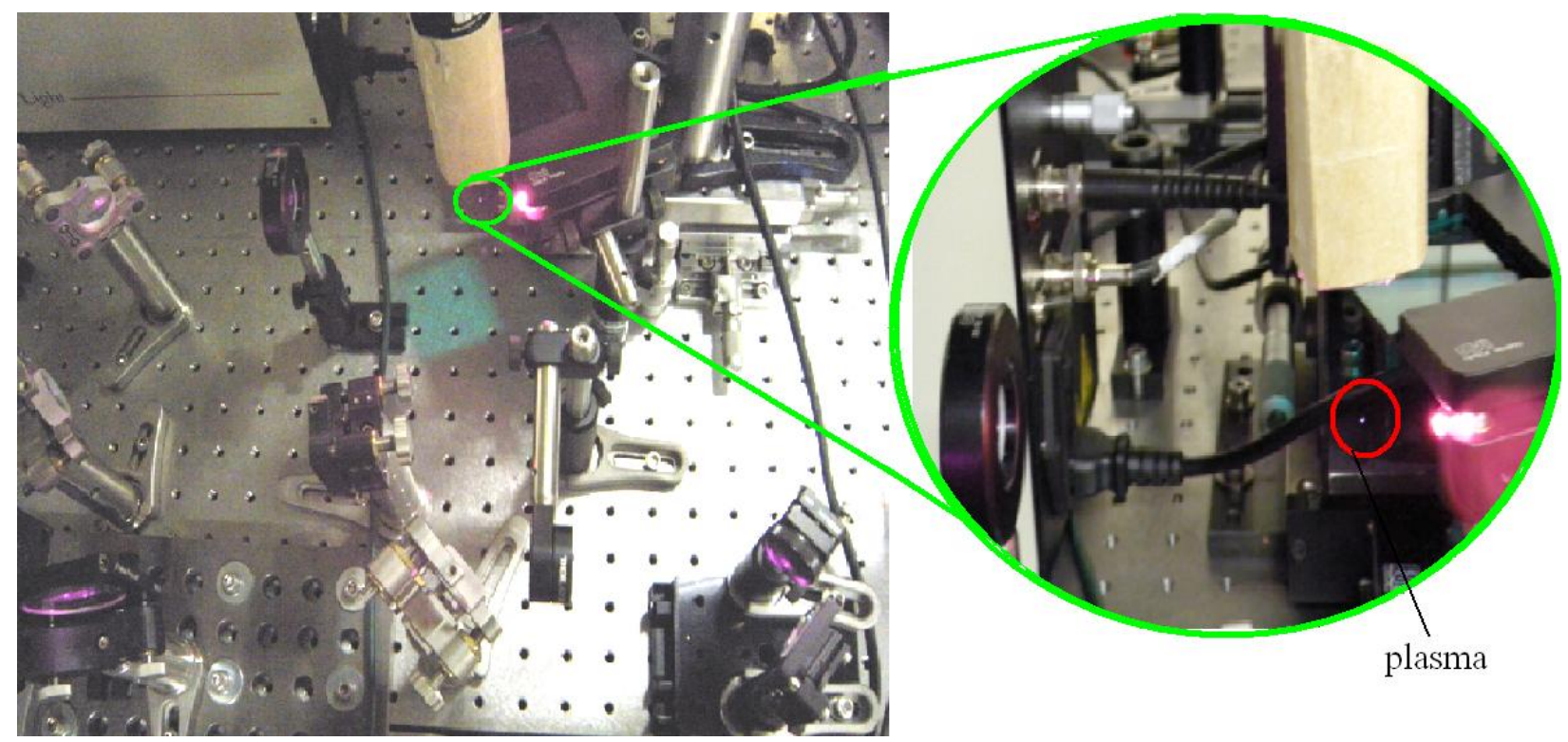

Figure 3. Photograph of plasma filamentation in the lab. 


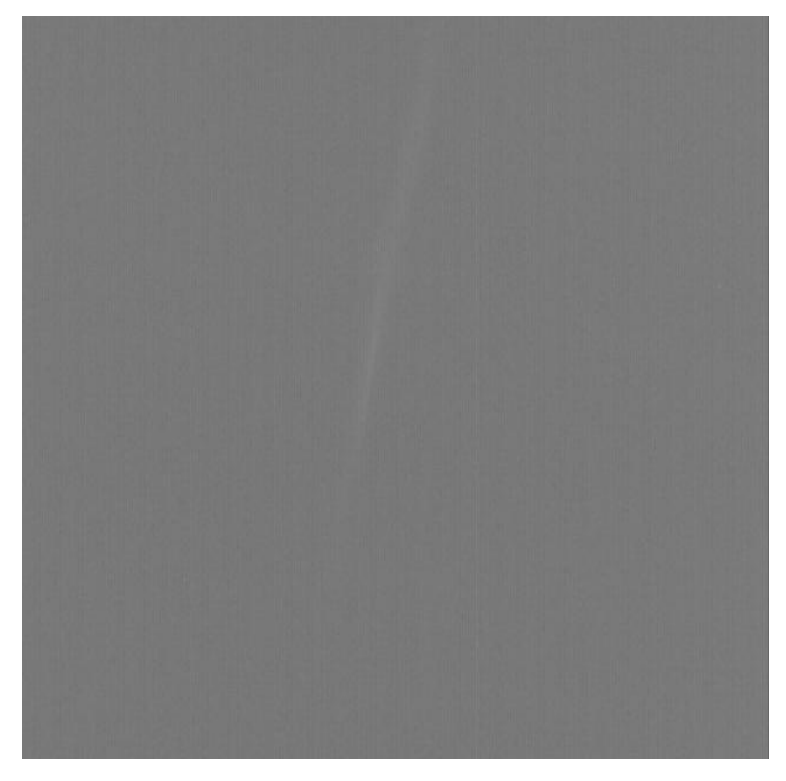

(a)

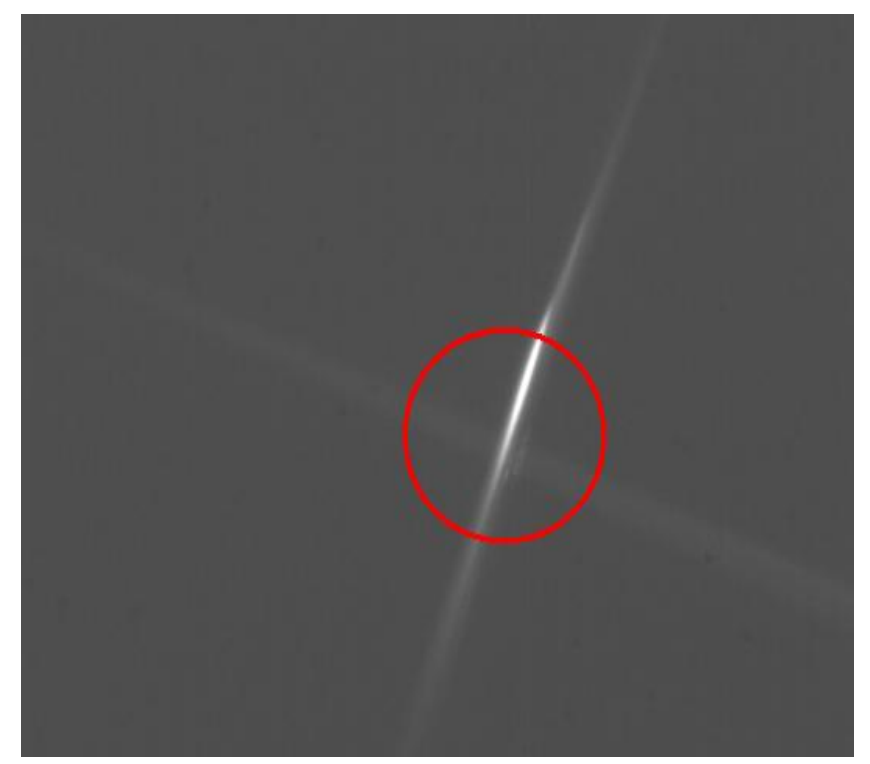

(b)

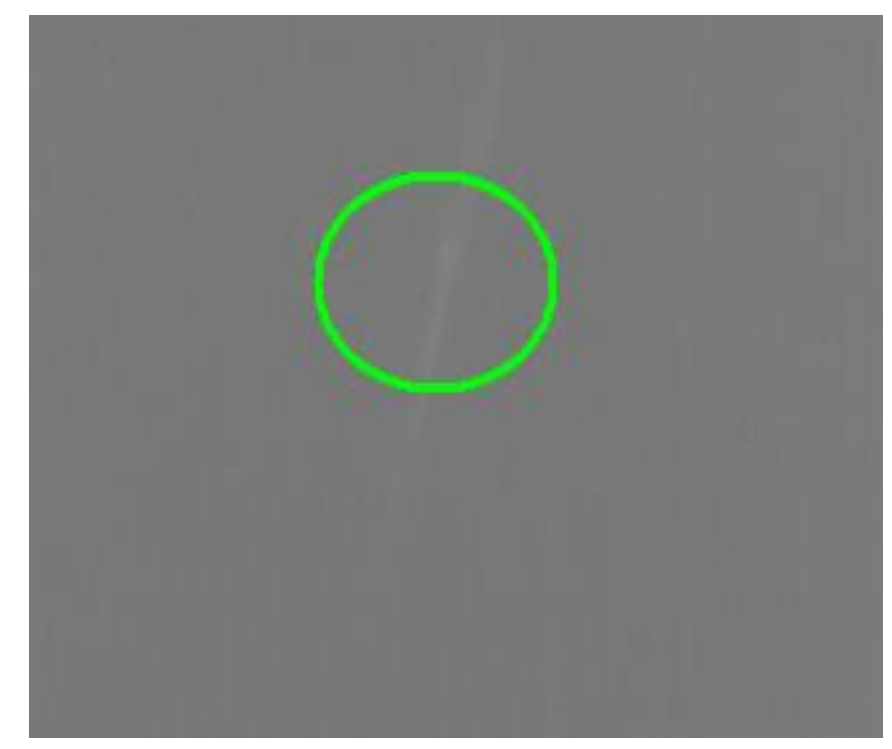

(c)

Figure 4: a) CCD camera image of plasma filaments before overlap. b) Image of plasma filaments intersecting at time-zero. Bright fluorescence is visible at region of overlap, as indicated in red. c) Image of plasma filaments at secondary maxima (after time-zero). Secondary fluorescence is indicated by green circle. 

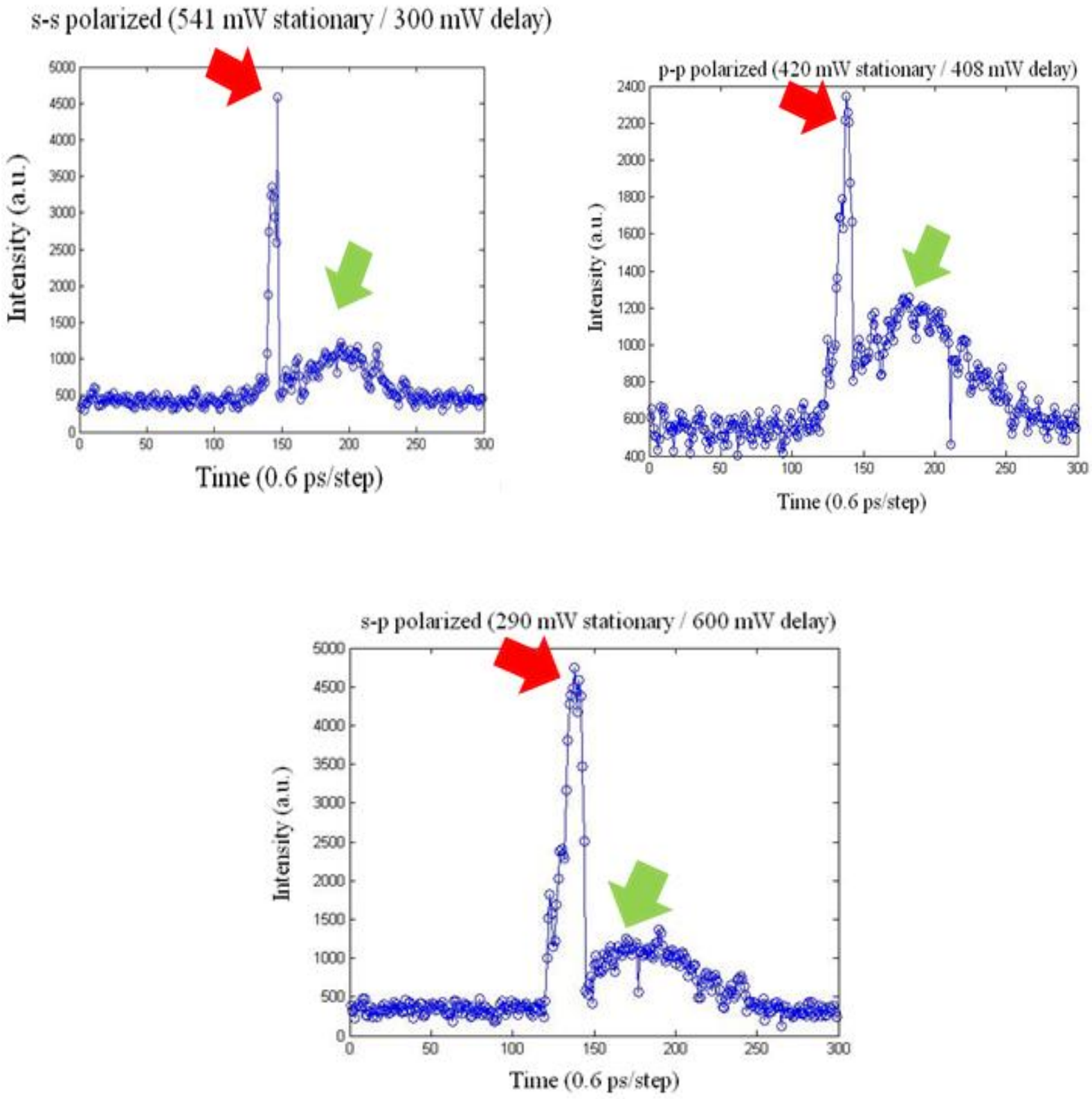

Figure 5. Peak Intensity vs. Time for three polarization sets. Peak intensity is indicated by the red arrow and secondary peak (also start of decay) is indicated by the green arrow. a) s-s polarization with $541 \mathrm{~mW}$ power in stationary arm (beam 2) and $300 \mathrm{~mW}$ power in delay arm (beam 1). b) p-p polarization with $420 \mathrm{~mW}$ power in stationary arm (beam 2) and $408 \mathrm{~mW}$ power in delay arm (beam 1). c) s-p polarization with $290 \mathrm{~mW}$ in stationary arm (beam 2) and $600 \mathrm{~mW}$ power in delay arm (beam 


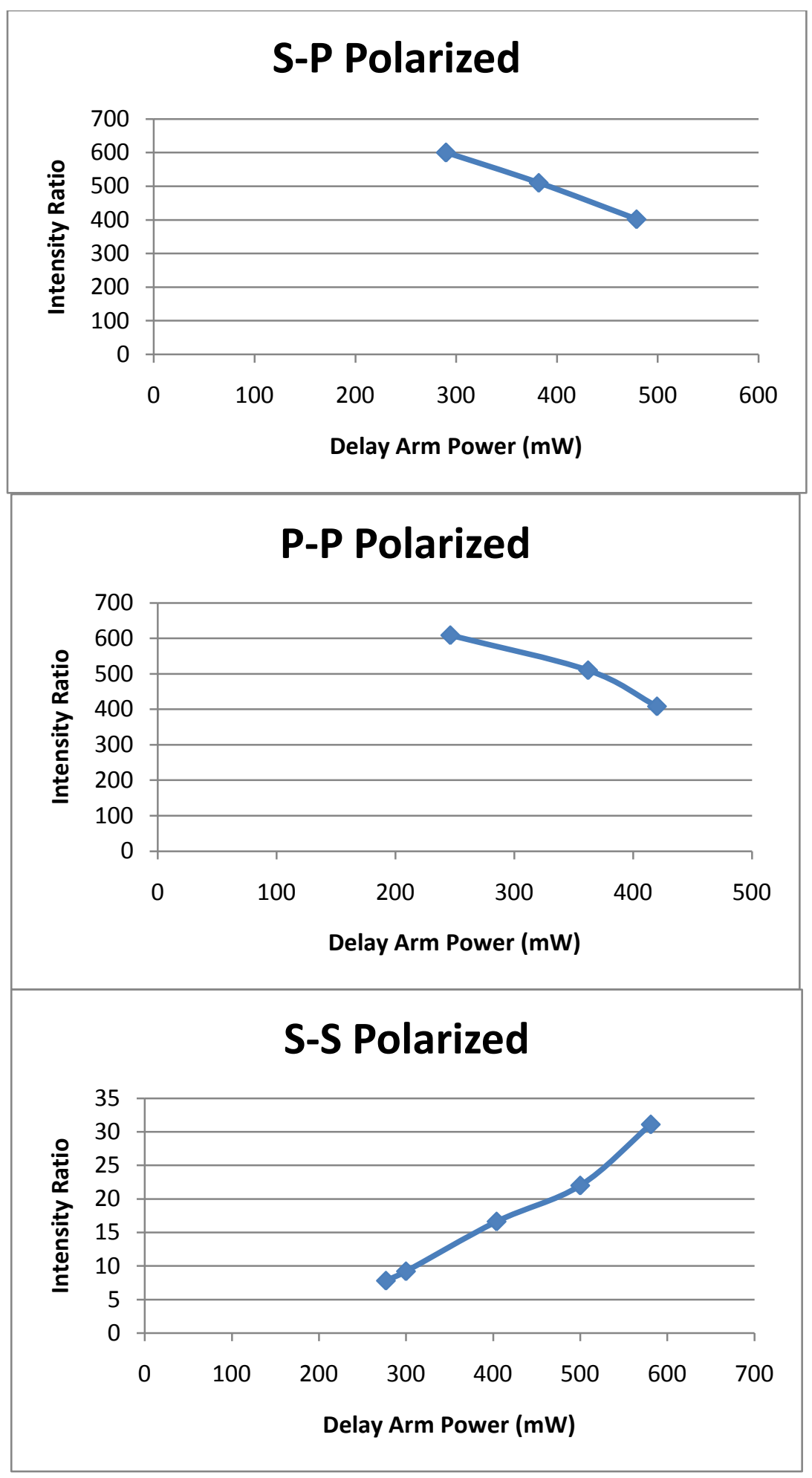

Figure 6: Ratio of peak intensity to background noise (intensity ratio) vs. power through the delay arm (beam 1) for all three polarization sets. Relationship seems to be linear. 


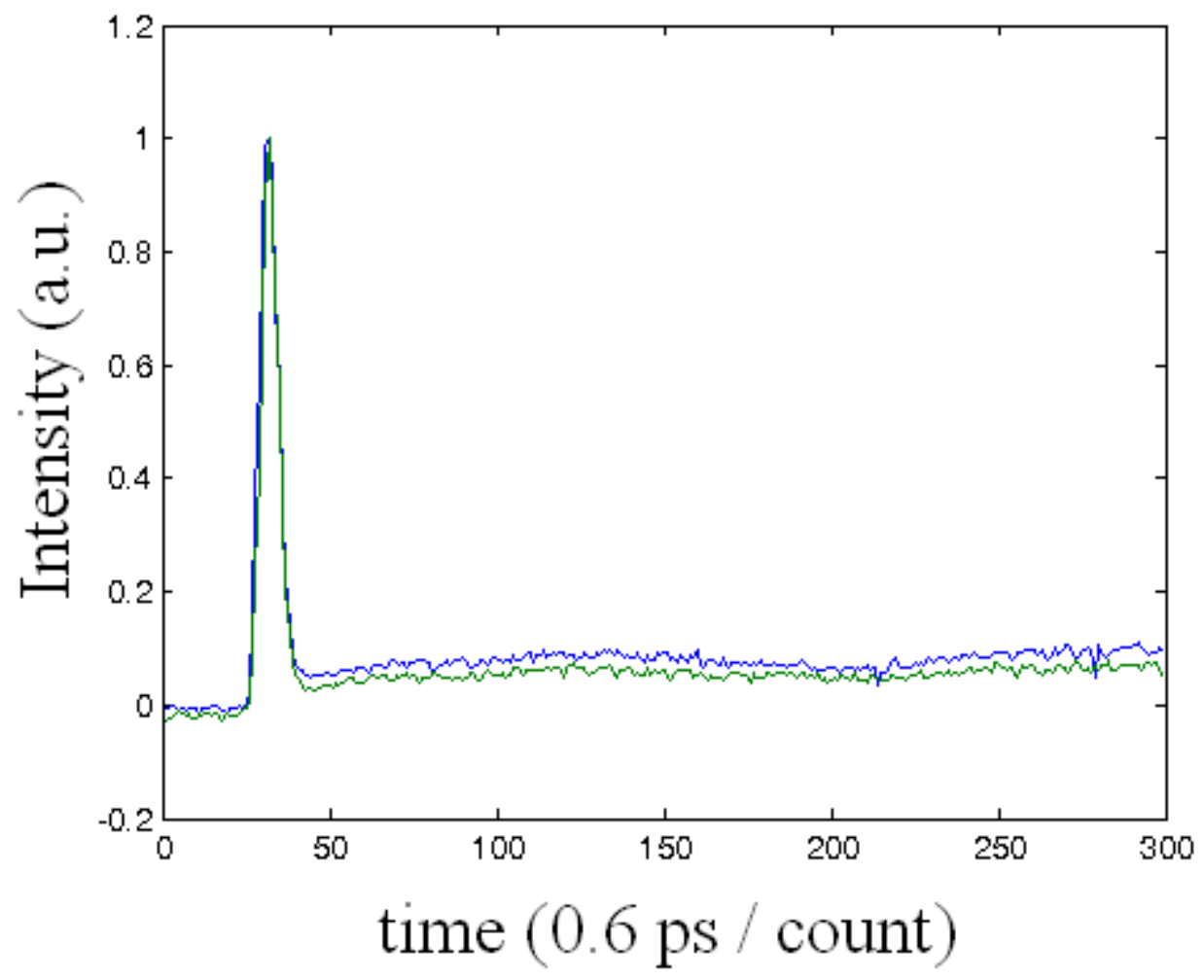

Figure 7: Normalized plot of intensity vs time for s-s polarization. Green trace corresponds to data taken with bluepass filter and blue trace corresponds to data taken without bluepass filter. Curves are normalized to the time zero peak. 


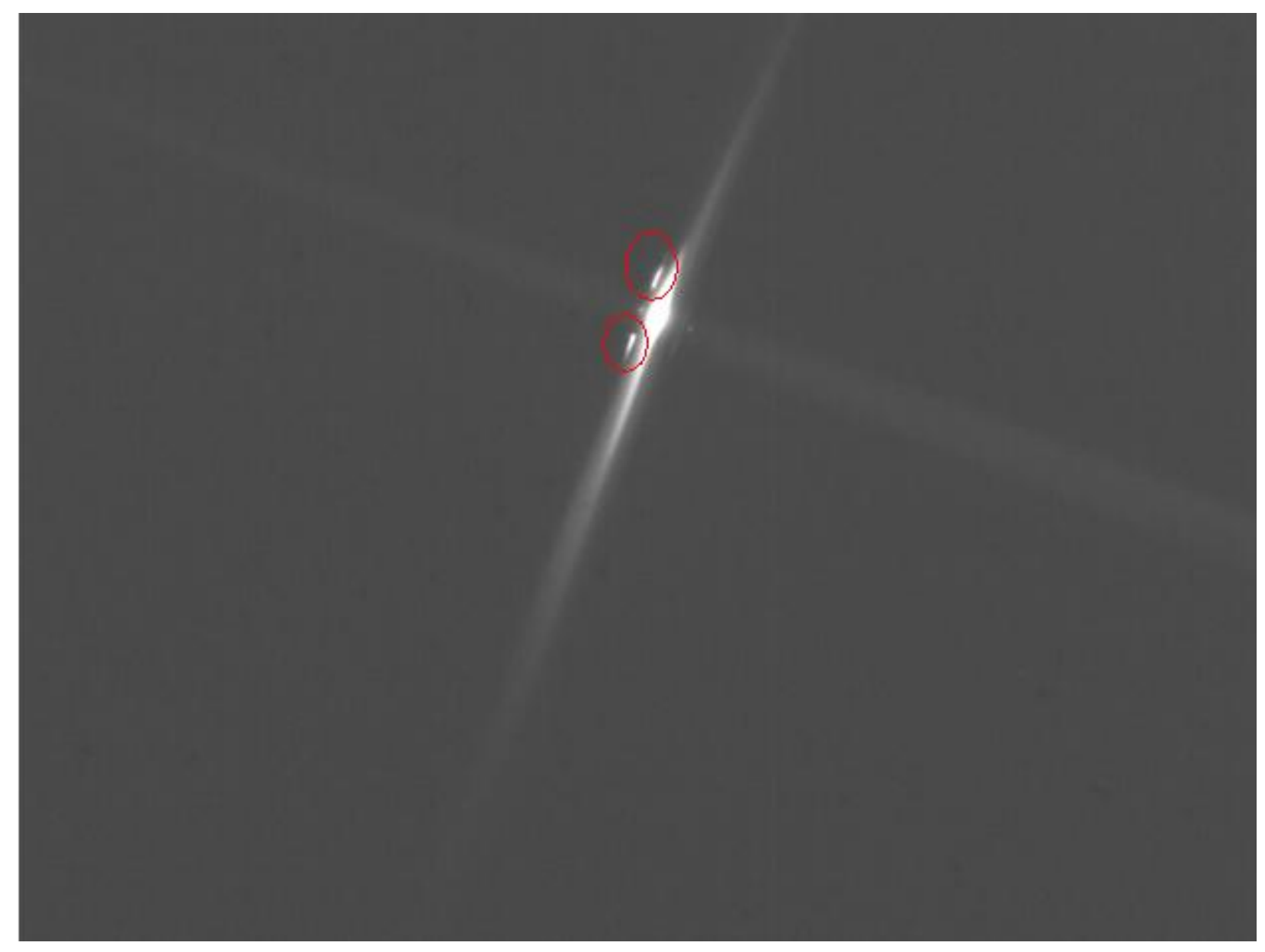

Figure 8: Secondary plasma formation as indicated by red circles. Effect is perhaps due to scattering. 

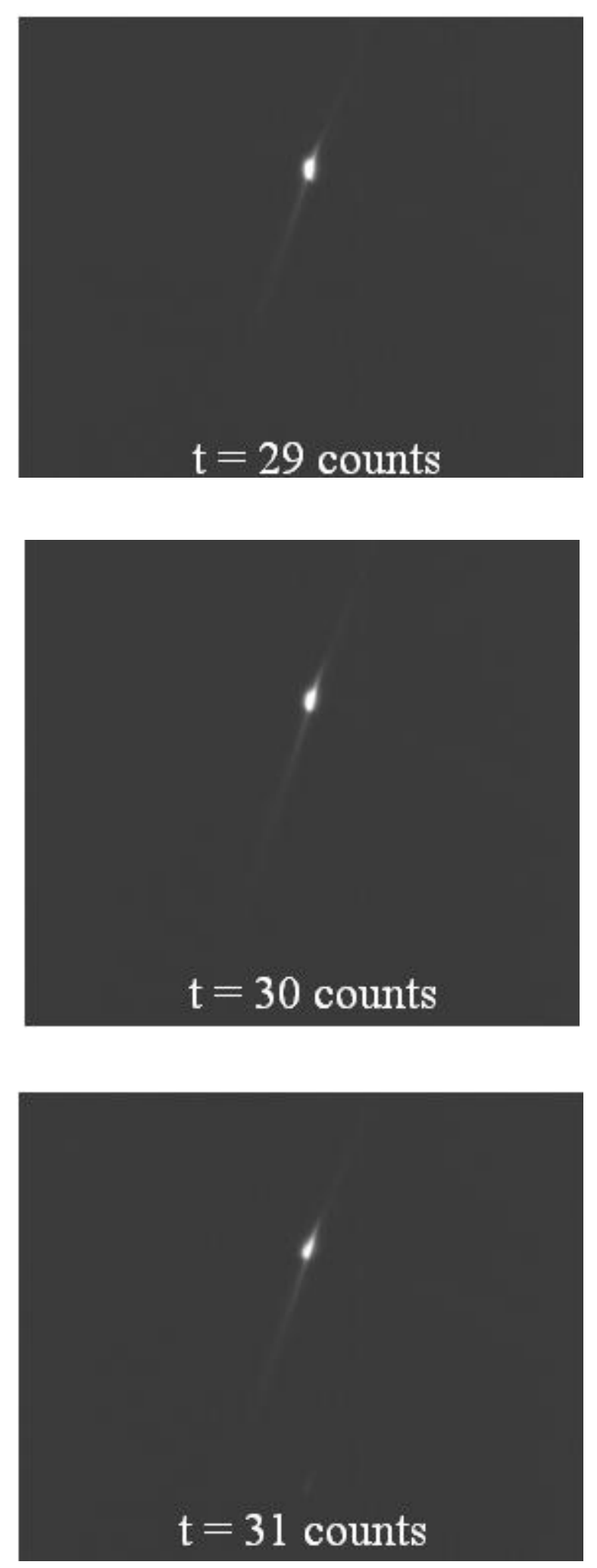

Figure 9: Diagonal rotation of overlap region through time. Plasma exhibits wavefront phenomenon providing an autocorrelation of the two pulses. 